\title{
Associations of ACE I/D and AGT M235T gene polymorphisms with the gestational hypertension and the fetal growth
}

\begin{abstract}
Hypertensive disease during pregnancy is a crucial perinatal problem. Preeclampsia is associated with complications for pregnant women and fetuses, so it is important to find causative factors. All over the world multiple researches are held among others on the subject of genetic conditioning of preeclampsia and gestational hypertension. Genes encoding the elements of the renin-angiotensin system, which regulates blood pressure, are the most commonly studied, especially the polymorphisms: ACE I/D and AGT M235T.

In this case-control study 168 pregnant patients were randomly enrolled from the patients hospitalized in the Perinatology and Obstetrics Department of the University Hospital in Cracow (Poland). In the study there were 104 normotensive pregnant women, 29 patients with preeclampsia and 35 patients with gestational hypertension. Genomic DNA was extracted from peripheral blood leukocytes and polymorphisms of ACE I/D and AGT M235T were genotyped from all patients. The genotypes distribution and allele frequencies of polymorphisms and its association to perinatal outcome in all groups were analyzed. A p-value $<0.05$ was considered as significant.

Clinical evaluation included the duration of the pregnancy, a method of the delivery, the birth weight, the birth length and the Apgar score. The risk of preeclampsia and gestational hypertension was evaluated. The CC genotype of AGT M235T polymorphism increases the risk of gestational hypertension. Genotype DD of the I/D polymorphism of the ACE gene is associated with the risk of lower birth weight percentile, but not with the increased risk of a fetal hypotrophy. The study shows the probable impact of ACE I/D and AGT M235T polymorphisms on pregnancy, but further studies on larger groups are needed.
\end{abstract}

Keywords: gestational hypertension, fetal growth restriction, preeclampsia, polymorphism, ACE I/D, AGT M235T
Special Issue - 2015

\author{
Hubert Huras, Krzysztof Rytlewski, \\ Katarzyna Kusmierska-Urban \\ Department of Obstetrics and Perinatology, Jagiellonian \\ University Medical College, Poland
}

Correspondence: Katarzyna Kusmierska-Urban, Department of Obstetrics and Perinatology, 23 Kopernika St, 3 I-50I Krakow, Poland,Tel +48 I2 42484 I2, Email k.kusmierska@gmail.com

Received: December 25, 20I4 | Published: March 9, 2015
Abbreviations: ACE I/D, angiotensin-converting enzyme insertion/deletion; AGT, angiotensynogen; RAS, reninangiotensin system; DNA, deoxyribonucleic acid; EDTA, ethylenediaminetetraacetic acid; PCR, polymerase chain reaction; RFLP, restriction fragment length polymorphism

\section{Introduction}

Hypertension during pregnancy is a heterogeneous group of a multisystemic disorders with elevated blood pressure with or without proteinuria. It is one of the most common pregnancy complication, responsible for high mortality and morbidity both of a mother and a child. ${ }^{1,2}$ In $10 \%$ to $15 \%$ preeclampsia and eclampsia are responsible for direct maternal deaths of pregnant women. ${ }^{3}$ There is no other effective treatment than finishing the pregnancy, as the expectant management of severe preeclampsia before 25 weeks of gestation is complicated with nearly $95 \%$ intrauterine fetal loss rate. ${ }^{4}$ All over the world multiple researches are held, among others, on the subject of genetic conditioning of preeclampsia and pregnancy induced hypertension. The heritable aspects of those conditions are complex. Although much information has been gathered, still the fully understanding of etiology is unknown. The usage of the identified risk factors of the disease is limited and in the stage of the clinic studies, what is connected with the complexity of the disease itself. Many variables, like environment, diet, style of life, socioeconomical conditions and stress has an impact on the progress as well as on the genetic expression of the disease. ${ }^{5}$ Currently it is believed that it is a multigenetic disease with great role of the epigenetic. It has been noticed that preeclampsia occurs more often among people who are related, which was the cause of significant suspicion of hereditary predisposition. ${ }^{6}$

Preeclampsia probably is associated with defective expression of endothelial adhesion factors and abnormal differentiation of the invasive cytotrophoblast. Interstitial implantation is mostly shallow, and, more importantly, the transluminal invasion is only fragmentary and it includes superficially located spiral arteries, which can lead to further functionally impaired flow in the maternal-placental circulation. ${ }^{8}$ The blood vessels do not undergo appropriate histological changes and remain high resistant. ${ }^{9}$ The low blood flow in the umbilical artery may also results in intrauterine growth retardation. ${ }^{10}$ This condition can lead to periodic reduction of blood flow to the placenta and, consequently, the repeated exposure to chronic hypoxia can cause the ischemia/reperfusion type of damage. ${ }^{11}$ It may further cause the activation of inflammatory cells such as the neutrophils and the release of cytotoxic oxygen free radicals. The impaired blood flow in the placenta is the first part of a two-stage model of preeclampsia etiopathogenesis, proposed in the Report of the Working Group NHLBI by Roberts et al. ${ }^{12}$

The second stage is the endothelial damage and disruption of the proper functioning (e.g. production of vasodilatory prostacyclin PGI2) and the related changes in the organs of the pregnant, like: kidney damage, liver, vascular circulatory system. Oxidative stress is likely to be the connecting element of the two stages. ${ }^{13}$ 
In normal pregnancy adaptive changes are observed in the cardiovascular system, including a reduction in systemic vascular resistance with subsequent lowering of blood pressure and an increase in blood volume. One of the mechanisms responsible for these changes is appropriate adaptation of the renin-angiotensin system (RAS-Renin-Angiotensin System).

Properly during pregnancy the blood levels of angiotensinogen, angiotensin II, aldosterone and plasma renin activity increase. Unfortunately, still insufficiently explored is the role of a local reninangiotensin system in utero-placental unit, which is influenced by both local and systemic blood circulating vasoactive substances. ${ }^{14}$

In pregnancy complicated by preeclampsia different levels of RAS activity were found and differences in the amounts of individual components, such as an increase in the concentration of molecular forms of angiotensinogen with an unchanged level of total angiotensinogen, a reduction in plasma renin activity, angiotensin II and aldosterone, ${ }^{15}$ and the raised value of serum angiotensinconverting enzyme. ${ }^{16}$ In preeclampsia there is a lower activity of the renin-angiotensin-aldosterone system compared to the normal pregnancy. ${ }^{17}$

The search for relevant genes is limited by incomplete understanding of the pathogenesis of the disease. Among many "genes candidates", which may have an impact on the origin of hypertension disease during pregnancy, there are those which products regulate the watersodium homeostasis, cardiovascular system and the blood pressure, like renin-angiotensin-aldosteron system, with angiotensinogen, angiotensin-converting enzyme and angiotensin receptors. One of the most commonly studied polymorphisms are: ACE I/D and AGT M235T.

The aims of this study were the evaluations of probable associations of ACE I/D and AGT-235 genes polymorphisms with the incidence of hypertension during pregnancy as well as their impact on perinatal outcome, especially on the fetal growth in polish population.

\section{Material and methods}

It is a case-controlled study with 168 pregnant patients randomly enrolled to the study from the patients hospitalized in the Perinatology and Obstetrics Department of the University Hospital in Cracow (Poland). The patients with hypertension during pregnancy were divided into two groups, one with preeclampsia in number of 29 patients and the second with gestational hypertension in number of 35 patients. The hypertension was considered, if the systolic/diastolic blood pressure was $\geq 140 / 90 \mathrm{mmHg}$ in two separate measurements. If the proteinuria in amount of $300 \mathrm{mg} / 1$ or $500 \mathrm{mg} / 24 \mathrm{~h}$ occurred with hypertension after the end of 20th week of pregnancy, preeclampsia was diagnosed.$^{18}$ The multiple pregnancy, stillbirth, fetal anomalies, patients with renal, liver or heart diseases as well as the withdrawal of patient's consent were the exclusion criteria from the study.

104 normotensive pregnant women were enrolled in the control group. Written informed consent was obtained from all the patients enrolled in this study and this study was approved by Ethic Committee of Jagiellonian University. Each patient was interviewed and detailed medical history, like gravidity and parity was taken. Clinical evaluation included the body mass index and the weight gain during the pregnancy, the duration of the pregnancy, a method of the delivery, the birth weight, the birth length and the Apgar score.
Samples of $5 \mathrm{ml}$ of venous blood from all patients were collected into EDTA tubes while performing standard medical procedures during hospitalization after the delivery. Blood samples were kept at $-80^{\circ} \mathrm{C}$ till all of the samples were gathered and analyzed at the same time. Genomic DNA was extracted from peripheral blood leukocytes. All the tested genetic polymorphisms were determined using the method of RFLP (Restriction Fragment Length Polymorphism). DNA isolation was performed with Kit High Pure PCR Template Preparation Kit. DNA quality and quantity were assessed on a spectrophotometer NanoDrop ND-1000. Genotyping was performed by investigators blinded to clinical status using the standardized protocols. ACE I/D polymorphism was detected with primers ACE-F: 5'-CTGGAGACCACTCCCATCCTTTCT-3' and ACE-R: 5'-GATGTGGCCATCACATTCGTCAGAT-3' while the AGT M235T with primers AGT-F: 5'-GAT GCG CAC AAG GTC CTG TC-3' and AGT-R: 5'- CAG GGT GCT GTC CAC ACT GGA CCC C- $3{ }^{3} \cdot{ }^{19-21}$

The characteristics of study groups were compared properly by Mann-Whitney test, Fisher's exact test, chi-square test, Classical analysis of variance and post-hoc test. The distribution of the genotype variants among studied subjects were compared by chisquared analysis and test Fisher's exact. Odds ratios were calculated as a measure of the association between the genotypes and clinical phenotypes. For each odds ratio, p values and $95 \%$ confidence intervals were calculated. A $p$ value of $<0.05$ was considered as statistically significant. The results were analyzed IBM SPSS Statistics v.20.

\section{Results and discussion}

The ACE I/D and AGT-235 polymorphisms genotype distribution and allele frequency were compatible with the Hardy-Weinberg equilibrium. In the first step the frequencies of genotypes in the groups were determined. The comparison of the prevalence of genotypes tested in each group showed no statistically significant differences. By studying the frequency of genotypes of AGT-235 gene it has been observed that the most common form observed in all groups was CT genotype. The homozygous CC occurred least often independently from the group (Table 1).

Table I Frequency of genotypes polymorphisms: I/D ACE and AGT-235

\begin{tabular}{|c|c|c|c|c|}
\hline & \multicolumn{3}{|l|}{ Study group } & \multirow[b]{2}{*}{$\mathbf{P}$} \\
\hline & $\begin{array}{l}\text { Gestational } \\
\text { hypertension } \\
{[n=35]}\end{array}$ & $\begin{array}{l}\text { Preeclampsia } \\
{[n=29]}\end{array}$ & $\begin{array}{l}\text { Control } \\
\text { group } \\
{[n=104]}\end{array}$ & \\
\hline \multicolumn{5}{|c|}{ I/D ACE } \\
\hline DD & $6(17,1 \%)$ & $8(27,6 \%)$ & $27(26,0 \%)$ & $\begin{array}{l}\mathrm{df}=4 \\
\mathrm{P}^{\mathrm{CHI}}=0,354\end{array}$ \\
\hline ID & $22(62,9 \%)$ & II (37,9\%) & $49(47,1 \%)$ & $P^{A}=0,439$ \\
\hline II & $7(20,0 \%)$ & $10(34,5 \%)$ & $28(26,9 \%)$ & $P^{A}=0,439$ \\
\hline \multicolumn{5}{|c|}{ AGT-235 } \\
\hline TT & $10(28,6 \%)$ & $8(27,6 \%)$ & $25(24,0 \%)$ & $\mathrm{PF}^{\mathrm{F}}=0,554$ \\
\hline CT & $20(57,1 \%)$ & $18(62,1 \%)$ & $72(69,2 \%)$ & $P^{A}=0,439$ \\
\hline $\mathrm{CC}$ & $5(14,3 \%)$ & $3(10,3 \%)$ & $7(6,7 \%)$ & $P^{A}=0,439$ \\
\hline
\end{tabular}

$\mathrm{Df}$, degrees of freedom; $\mathrm{CHI}$, chi-square test; $\mathrm{F}$-test, fisher's exact; $\mathrm{A}$, classical analysis of variance 
Then it was determined whether the presence of tested genotypes was associated with the risk of gestational hypertension or preeclampsia. For this purpose a logistic regression model was used, taking into account the one-dimensional model and multidimensional models, which enabled the standardization of the potential confounding variables.

Comparison of I/D polymorphism of the ACE gene revealed no significant differences between the two groups and the control group for the risk of preeclampsia or gestational hypertension (Table 2) (Table 3). The analysis of the risk of preeclampsia did not show significant impact of the AGT gene M235T polymorphism (Table
2). In contrast, it has been shown that the presence of homozygous $\mathrm{CC}$ significantly increases the risk of gestational hypertension in the multivariate model standardized for confounders (Table 3).

The relationship of the prevalence of specific genotypes with the duration of pregnancy and newborn data, namely: birth weight, the neonate's body length, birth weight percentile and the results of the Apgar score were analyzed. We analyzed the number of newborns whose condition was rated less than 7 points according to the Apgar score. Such children prevailed bit in the DD genotype of the ACE gene group and CC genotype of the AGT-235 gene. None of the observed differences were statistically significant (data not shown).

Table 2 The risk of preeclampsia depending on the genotypes: I/D ACE and AGT-235

\begin{tabular}{|c|c|c|c|c|c|c|}
\hline & $O R^{\prime}(95 \% \mathrm{Cl})$ & $\mathbf{P}$ & $\mathrm{OR}^{2}(95 \% \mathrm{Cl})$ & $\mathbf{P}$ & $O R^{3}(95 \% \mathrm{Cl})$ & $\mathbf{P}$ \\
\hline \multicolumn{7}{|c|}{ I/D ACE } \\
\hline DD & 1 & & 1 & & 1 & \\
\hline ID & $0,76(0,27-2, I I)$ & 0,59 & $0,64(0,2 I-I, 97)$ & 0,43 & $0,68(0,22-2,14)$ & 0,51 \\
\hline II & $\mathrm{I}, 2 \mathrm{I}(0,4 \mathrm{I}-3,5 \mathrm{I})$ & 0,73 & I,47 (0,44-4,94) & 0,53 & $\mathrm{I}, 6 \mathrm{I}(0,47-5,5 \mathrm{I})$ & 0,45 \\
\hline \multicolumn{7}{|c|}{ AGT-235 } \\
\hline TT & 1 & & I & & I & \\
\hline CT & $0,78(0,30-2,02)$ & 0,61 & $0,70(0,26-I, 9 I)$ & 0,49 & $0,62(0,22-I, 72)$ & 0,36 \\
\hline $\mathrm{CC}$ & $\mathrm{I}, 34(0,28-6,43)$ & 0,72 & $2,76(0,14-54,8)$ & 0,51 & $2,79(0,14-56,4)$ & 0,50 \\
\hline
\end{tabular}

OR, odds ratio; $\mathrm{Cl}$, confidence interval

logistic regression model: I - dimensional model; 2 - model multidimensional, standardized for maternal age, the incidence of miscarriages in the past, maternal BMI in early pregnancy; 3 - model multidimensional, standardized variables in model 2, diabetes diagnosis (yes / no), hypothyroidism (yes / no), other concomitant diseases (yes / no).

Table 3 The risk of gestational hypertension depending on the genotypes: I/D ACE and AGT-235

\begin{tabular}{|c|c|c|c|c|c|c|}
\hline & OR' (95\% CI) & $\mathbf{P}$ & $\mathrm{OR}^{2}(95 \% \mathrm{CI})$ & $\mathbf{P}$ & $\mathrm{OR}^{3}(95 \% \mathrm{CI})$ & $\mathbf{P}$ \\
\hline \multicolumn{7}{|c|}{ I/D ACE } \\
\hline DD & 1 & & I & & I & \\
\hline ID & $2,02(9,73-5,59)$ & 0,18 & I,96 (0,6I-6,29) & 0,26 & I,98 (0,59-6,68) & 0,27 \\
\hline II & I,I $3(0,34-3,78)$ & 0,85 & $\mathrm{I}, 45(0,37-5,74)$ & 0,60 & I,44 (0,33-6,26) & 0,63 \\
\hline \multicolumn{7}{|c|}{ AGT-235 } \\
\hline TT & $\mathrm{I}$ & & I & & I & \\
\hline $\mathrm{CT}$ & $0,70(0,29-1,69)$ & 0,42 & $0,98(0,34-2,79)$ & 0,97 & $0,94(0,32-2,77)$ & 0,91 \\
\hline $\mathrm{CC}$ & I,79 (0,46-6,97) & 0,40 & $12,9(1,23-136,9)$ & 0,03 & II,4 (I,03-I26,7) & 0,047 \\
\hline
\end{tabular}

OR, odds ratio; $\mathrm{Cl}$, confidence interval

Logistic regression model, I - dimensional model; 2 - model multidimensional, standardized for maternal age, the incidence of miscarriages in the past, maternal BMI in early pregnancy; 3 - model multidimensional, standardized variables in model 2, diabetes diagnosis (yes / not), hypothyroidism (yes / no), other concomitant diseases (yes / no).

By examining differences within the ACE gene genotypes it was observed that the children varied with the birth weight percentile. Children with DD genotype had significantly lower percentile values than the ID genotype group $(p<0.05)$. The similar differences in birth weight were observed, but they did not reach statistical significance $(\mathrm{p}=0,076)$ (Table 4).
While analyzing the risk of intrauterine growth restriction depending on the ACE gene it has been observed no increased risk of having a child with hypotrophy $<10$ th percentile, after standardization for age of the mother, the occurrence of miscarriage in the past and BMI of the mother at the beginning of pregnancy (Table 5). 
Table 4 Parameters of the newborn depending on the genotypes of I/D ACE

\begin{tabular}{|c|c|c|c|c|}
\hline & \multicolumn{3}{|c|}{ The genotypes of the I/D ACE gene } & \multirow[b]{2}{*}{$\mathbf{P}$} \\
\hline & $\begin{array}{l}\text { DD } \\
{[n=4 I]}\end{array}$ & ID $[n=82]$ & II $[n=45]$ & \\
\hline \multicolumn{5}{|c|}{ Duration of pregnancy [week] } \\
\hline Mean [SD] & $37,9[3,4]$ & $38,6(3,0)$ & $38,3(2,8)$ & $P^{A}=0,467$ \\
\hline Min -max & $26-42$ & $26-42$ & $27-42$ & \\
\hline \multicolumn{5}{|c|}{ Birth weight [g] } \\
\hline Mean [SD] & 2896 [896] & $3240[8 \mathrm{II}]$ & $3123[774]$ & $P^{A}=0,076$ \\
\hline Min -max & $590-4170$ & $720-4430$ & $700-4160$ & \\
\hline \multicolumn{5}{|c|}{ Length of newborn $[\mathrm{cm}]$} \\
\hline Mean [SD] & $52,1[6,5]$ & $53,9[5,5]$ & $52,9[5,3]$ & $P^{A}=0,187$ \\
\hline Min -max & $30-62$ & $33-62$ & $33-62$ & \\
\hline \multicolumn{5}{|c|}{ Percentile of birth weight } \\
\hline Mean [SD] & $27,6[23,7]$ & $43,0[30,1]$ & $39,3[28,2]$ & $P^{A}=0,013$ \\
\hline Min -max & $0,5-88,0$ & I,0-99,0 & $0,5-95,0$ & DD-ID* \\
\hline \multicolumn{5}{|c|}{ Apgar in I min } \\
\hline Mean [SD] & $9,4[1,2]$ & $9,5[\mathrm{I}, \mathrm{I}]$ & $9,4[1,5]$ & $P^{A}=0,756$ \\
\hline Min -max & $6-10$ & $4-10$ & $3-10$ & \\
\hline
\end{tabular}

A, classical analysis of variance; ${ }^{*} \mathrm{p}<0,05$ post hoc test

Table 5 The risk of hypotrophy ( $<10$ percentyle) depending on the I/D ACE genotype

\begin{tabular}{|c|c|c|c|c|c|}
\hline $\begin{array}{l}\text { ORI (95\% } \\
\text { CI) }\end{array}$ & $\mathbf{P}$ & $\begin{array}{l}\text { OR2 }(95 \% \\
\text { Cl) }\end{array}$ & $\mathbf{P}$ & $\begin{array}{l}\text { OR3 (95\% } \\
\mathrm{Cl})\end{array}$ & $\mathbf{p}$ \\
\hline
\end{tabular}

I/D ACE

\begin{tabular}{|c|c|c|c|c|c|c|}
\hline DD & 1 & & 1 & & I & \\
\hline ID & $\begin{array}{l}0,65(0,29- \\
\mathrm{I}, 49)\end{array}$ & 0,31 & $\begin{array}{l}0,82(0,32- \\
2,09)\end{array}$ & 0,68 & $\begin{array}{l}1,04(0,37- \\
2,93)\end{array}$ & 0,93 \\
\hline II & $\begin{array}{l}0,67(0,26- \\
I, 72)\end{array}$ & 0,40 & $\begin{array}{l}0,96(0,33- \\
2,83)\end{array}$ & 0,94 & $\begin{array}{l}0,87(0,26- \\
2,88)\end{array}$ & 0,82 \\
\hline
\end{tabular}

OR, odds ratio

Logistic regression model, I-dimensional model, 2-model multi-dimensional, standardized for maternal age, the incidence of miscarriages in the past, BMI mothers in early pregnancy; 3-model multi-dimensional, standardized variables of model 2 and the average values of pressure, the presence of proteinuria.

The interpretation of data on the genetic background of gestational hypertension or preeclampsia is very difficult, because there are often contradictory results of case-control studies, which results often fail to be repeated in subsequent studies. Part of the differences stem from the multifactorial ground of discussed diseases, and some of them may be a consequence of inconsistent patients' inclusion criteria in the study, definition or statistical analysis of the data. Preeclampsia is also a disease with a variable phenotype.

The polymorphism of the ACE gene is a deletion (allele D) or insertion (allele I), repetitive Alu nucleotide sequence of about $287 \mathrm{bp}$ intron 16 and affects the amount of enzyme in serum. Subjects with a deletion show a higher enzyme concentration than subjects with the insertion. What is more, each allele exhibits an additive effect of the level of ACE and therefore are co-dominants. ${ }^{19,22}$ Insertion allele has a sequence similar to silencer sequence, which may explain why the D allele corresponds to the higher levels of the enzyme than the allele I. ${ }^{23}$

Extremely interesting is the work of Mello et al., based on which it is concluded that the polymorphism ACE I/D has an effect on the circulation in utero-placental and fetal unit. There was clearly increased pulsatility index in both the umbilical artery and in the uterine arteries in the pregnant carriers of DD genotype compared to genotype II. Moreover, statistically significant differences were observed between genotypes.In the DD group were found higher fetal growth restriction rate, earlier gestational age at delivery and lower birth weight of children. ${ }^{24}$ These results are in compliance with the results of the present work, but without reaching the threshold of statistical significance. This indicates the role of ACE polymorphism in the abnormal development of uteroplacental circulation, what potentially can initiate the cascade of events that leads to adverse perinatal outcome like hypertension or has an impact on the fetal growth.

In one study the Thr235/Thr235 of AGT and DD of the ACE genotypes were significantly associated with preeclampsia, ${ }^{25}$ which stays in the disagreement with the results our study. The results of this study are consistent with the different study on the Polish population, ${ }^{26}$ where there was no difference in allele frequency I/D ACE gene between the control group and the group with gestational hypertension or research on the Korean population ${ }^{27}$ which did not confirm the role of the I/D polymorphism in preeclampsia.

A single nucleotide polymorphism M235T of AGT gene located in exon 2 in region $3^{\prime}$ leads to the conversion of cytosine by thymine at position 704, which results in substitution of a threonine in place of methionine at aminoacid chain at position 235. It is one of the most frequently studied polymorphisms of this gene, especially in the context of hypertension. ${ }^{28} 235 \mathrm{~T}$ (allel C) of AGT gene correlates with elevated levels of AGT in the blood, ${ }^{29}$ which also showed higher concentrations of AGT levels in heterozygotes 235MT compared to homozygous 235MM. ${ }^{30,31}$ Another meta-analysis found that 235T homozygotes have not only higher levels of AGT in the blood, but also about $11 \%$ higher relative risk of hypertension compared to homozygotes $235 \mathrm{M} .^{32}$

This polymorphism, which has been associated with big hopes, as a causative factor or a marker of hypertensive disorders in pregnancy, unfortunately, did not bring definitive answers. Although a Dutch study clearly demonstrated a higher incidence of complications such as gestational hypertension and preeclampsia in women TT carriers, so in $\mathrm{CC}$ homozygotes $(\mathrm{OR}=1.43,95 \% \mathrm{CI}=1.02-2.01, \mathrm{p}=0.04),{ }^{33}$ what stays in agreement with our results, other studies deny that association. ${ }^{34}$

Because in the open literature are many often conflicting results concerning these polymorphisms, it seems necessary to continue research on defining their role in hypertension in pregnancy and their effect on the perinatal outcome.

\section{Conclusion}

On the basis of this work, it can be concluded, that the CC genotype of AGT M235T polymorphism increases the risk of gestational hypertension. 
Genotype DD of the I/D polymorphism of the ACE gene is associated with the risk of lower birth weight percentile, but not with the increased risk of a fetal hypotrophy. More studies on larger groups are needed.

\section{Acknowledgments}

This research was supported by funding from Jagiellonian University Medical College.

\section{Conflict of interest}

The authors declare that there is no conflict of interest.

\section{References}

1. Langenveld J, Ravelli AC, van Kaam AH, et al. Neonatal outcome of pregnancies complicated by hypertensive disorders between 34 and 37 weeks of gestation: a 7 year retrospective analysis of a national registry. Am J Obstet Gynecol. 2011;205(6):540.e1-540.e7.

2. Aali BS, Ghafoorian J, Mohamad-Alizadeh S. Severe preeclampsia and eclampsia in Kerman, Iran: complications and outcomes. Med Sci Monit. 2004;10(4):CR163-CR167.

3. Duley L. The global impact of pre-eclampsia and eclampsia. Semin Perinatol. 2009;33(3):130-137.

4. Sezik M, Ozkaya O, Toyran Sezik H, et al. Expectant management of severe preeclampsia presenting before 25 weeks of gestation. Med Sci Monit. 2007;13(11):CR523-CR537.

5. Williams PJ, Morgan L. The role of genetics in pre-eclampsia and potential pharmacogenomic interventions. Pharmgenomics Pers Med. 2012;5:37-51

6. Cnattingius S, Reilly M, Pawitan Y, et al. Maternal and fetal genetic factors account for most of familial aggregation of preeclampsia: a populationbased Swedish cohort study. Am J Med Genet A. 2004;130A(4):365-371.

7. McMaster MT, Zhou Y, Fisher S. Abnormal placentation and the syndrome of preeclampsia. Semin Nephrol. 2004;24(6):540-547.

8. Meekins JW, Pijnenborg R, Hanssens M, et al. A study of placental bed spiral arteries and trophoblast invasion in normal and severe preeclamptic pregnancies. Br J Obstet Gynaecol. 1994;101(8):669-674.

9. Zhou Y, Damsky CH, Fisher SJ. Preeclampsia is associated with failure of human cytotrophoblasts to mimic a vascular adhesion phenotype. One cause of defective endovascular invasion in this syndrome? Clin Invest 1997;99(9):2152-2164.

10. Rytlewski K, Zdebski Z, Kalita J. Measurement of blood flow in the umbilical artery of women with pregnancy complications. Ginekol Pol. 1989;60(4):197-201

11. Hung TH, Skepper JN, Burton G. In vitro ischemia-reperfusion injury in term human placenta as a model for oxidative stress in pathological pregnancies. Am J Pathol. 2001;159(3):1031-1043.

12. Roberts JM, Pearson G, Cutler J, et al. Summary of the NHLBI Working Group on Research on Hypertension During Pregnancy. Hypertension. 2003;41(3):437-445.

13. Roberts JM, Hubel CA. Is oxidative stress the link in the two-stage model of pre-eclampsia? Lancet. 1999;354(9181):788-789.

14. Poisner AM. The human placental renin-angiotensin system. Front Neuroendocrinol. 1998;19(3):232-252.

15. Brown MA, Wang J, Whitworth JA. The rennin-angiotensin-aldosterone system in pre-eclampsia. Clin Exp Hypertens. 1997;19(5-6):713-726.
16. Merrill DC, Karoly M, Chen K, et al. Angiotensin-(1-7) in normal and preeclamptic pregnancy. Endocrine. 2002;18(3):239-245.

17. Langer B, Grima M, Coquard C, et al. Plasma active renin, angiotensin $\mathrm{I}$, and angiotensin II during pregnancy and in preeclampsia. Obstet Gynecol. 1998;91(2):196-202.

18. Lindheimer MD, Taler SJ, Cunningham FG, American Society of Hypertension. ASH position paper: hypertension in pregnancy. J Clin Hypertens (Greenwich). 2009;11(4):214-225.

19. Rigat B, Hubert C, Corvol P, et al. PCR detection of the insertion/deletion polymorphism of the human angiotensin converting enzyme gene (DCP1) (dipeptidyl carboxypeptidase 1). Nucleic Acids Res. 1992;20(6):1433.

20. Niu T, Yang J, Wang B, et al. Angiotensinogen gene polymorphisms M235T/T174M: no excess transmission to hypertensive Chinese. Hypertension. 1999;33(2):698-702.

21. Olsson M, Annerbrink K, Westberg L, et al. Angiotensin-related genes in patients with panic disorder. Am J Med Genet B Neuropsychiatr Genet. 2004;127B(1):81-84.

22. Tiret L, Rigat B, Visvikis S, et al. Evidence, from combined segregation and linkage analysis, that a variant of the angiotensin I-converting enzyme (ACE) gene controls plasma ACE levels. Am J Hum Genet. 1992;51(1):197-205.

23. Jackson A, Brown K, Langdown J, et al. Effect of the angiotensinconverting enzyme gene deletion polymorphism on the risk of venous thromboembolism. Br J Haematol. 2000;111(2):562-564.

24. Mello G, Parretti E, Gensini F, et al. Maternal-fetal flow, negative events, and preeclampsia: role of ACE I/D polymorphism. Hypertension. 2003;41(4):932-937.

25. Procopciuc LM, Caracostea G, Zaharie G, et al. Maternal/newborn genotype contribution of the renin-angiotensin system (Met235Thr, Thr174Met, I/D-ACE, A2350G-ACE, A1166C-AT2R1, C3123AAT2R2, 83A/G-REN) to the risk of pre-eclampsia: a Romanian study. $J$ Renin Angiotensin Aldosterone Syst. 2011;12(4):539-548.

26. Nalogowska-Glosnicka K, Lacka BI, Zychma MJ, et al. Angiotensin II type 1 receptor gene A1166C polymorphism is associated with the increased risk of pregnancy-induced hypertension. Med Sci Monit. 2000;6(3):523-529.

27. Kim YJ, Park MH, Park HS, et al. Associations of polymorphisms of the angiotensinogen M235 polymorphism and angiotensin-convertingenzyme intron 16 insertion/deletion polymorphism with preeclampsia in Korean women. Eur J Obstet Gynecol Reprod Biol. 2004;116(1):48-53.

28. Inoue I, Nakajima T, Williams CS, et al. A nucleotide substitution in the promoter of human angiotensinogen is associated with essential hypertension and affects basal transcription in vitro. $J$ Clin Invest. 1997;99(7):1786-1797.

29. Aggarwal S, Dimri N, Tandon I, et al. Preeclampsia in North Indian women: the contribution of genetic polymorphisms. J Obstet Gynaecol Res. 2011;37(10):1335-1341.

30. Bloem LJ, Foroud TM, Ambrosius WT, et al. Association of the angiotensinogen gene to serum angiotensinogen in blacks and whites. Hypertension. 1997;29(5):1078-1082.

31. Jeunemaitre X, Soubrier F, Kotelevtsev YV, et al. Molecular basis of human hypertension: role of angiotensinogen. Cell. 1992;71(1):169-180.

32. Sethi AA, Nordestgaard BG, Tybjaerg-Hansen A. Angiotensinogen gene polymorphism, plasma angiotensinogen, and risk of hypertension and ischemic heart disease: a meta-analysis. Arterioscler Thromb Vasc Biol. 2003;23(7):1269-1275. 
33. Zafarmand $\mathrm{MH}$, Franx A, Sabour S, et al. The M235T variant of the angiotensinogen gene is related to development of self-reported hypertension during pregnancy: the Prospect-EPIC cohort study. Hypertens Res. 2008;31(7):1299-1305.
34. Suzuki Y, Tanemura M, Suzuki Y, et al. Is angiotensinogen gene polymorphism associated with hypertension in pregnancy? Hypertens Pregnancy. 1999;18(3):261-271. 